\title{
Omnidirectional Reflection Band in Thue-morse Quasicrystal Containing Indefinite Metamaterials
}

\author{
Yong-Qiang KANG ${ }^{1,2, a, *}$ \\ ${ }^{1}$ Institute of solid state physics, Shanxi Datong University, Datong, Shanxi 037009, China \\ ${ }^{2}$ Institute of Space optics, School of Science, Xi'an Jiaotong University, Xi'an 710049, China \\ akyq_2000@sohu.com \\ ${ }^{*}$ Corresponding author
}

Keywords: Electromagnetic Optics, Omnidirectional Reflection Band, Indefinite Metamaterials.

\begin{abstract}
By means of transfer matrix method, the reflectance of Thue-Morse (T-M) structure containing indefinite metamaterials is investigated It is shown that there is an omnidirectional reflectance band (ORB), and location of the ORB remains invariant with the change of generation order. The width of the ORB is determined by the higher frequency band edge for TE polarization and the lower frequency band edge for TM polarization. The location and width of the ORB is not affected by lattice scaling factor. When an impurity is introduced, a defect mode appears in the band gap. The position of the defect modes is weakly dependent on incident angle for TE polarization, but is affected by incident angle for TM polarization.
\end{abstract}

\section{Introduction}

Indefinite metamaterials (IMMs), a kind of artificial materials, have recently received increasing attention $^{[1,2,3]}$. It is one of the anisotropic metamaterials, whose principal components of the electric permittivity and magnetic permeability tensors are not all of the same sign. Since only a part of the constitutive parameters is required to be negative, compared with the isotropic left-handed materials (LHMs), IMMs are much easier to be fabricated. Under certain conditions, the IMMs can take the place of the isotropic structures, and be used as a substitute for LHMs to exhibit a variety of unusual effects, including negative refraction and backward-wave effects, the anticutoff or never-cutoff effects, near-field focusing, anomalous total reflection phenomenon, and so on ${ }^{[4-6]}$. In addition, one dimensional photonic crystals (1DPCs) containing IMMs can result in a similar zero average index (zero- $\bar{n}$ ) gap usually appearing in 1DPC containing isotropic left-handed materials ${ }^{[7-10]}$.

Quasicrystals are some kind of non-periodic structures which lack long-range translational symmetry, but possess a certain orientation order. The structural ordering of quasicrystals is between the periodic and disordered systems ${ }^{[1]}$. Two well-known examples of one dimensional quasicrystal are the Fibonacci and Thue-Morse (T-M) structures. The dielectric T-M systems may exhibit fractal gap, in case fractal structures are involve ${ }^{[12]}$. The scaling of fractal gaps occurring in dielectric T-M multilayer provides a large omnidirectional band gap ${ }^{[13,14]}$. Recently, the omnidirectional reflection bands of T-M quasicrystals with single-negative materials have been presented ${ }^{[15]}$. However, to the best of my knowledge, the T-M quasicrystals with indefinite metamaterials have not been reported.

In this paper, the reflection bands in T-M quasicrystals with indefinite metamaterials are investigated theoretically. It is shown that there is a new type of ORB exists in the T-M structure with indefinite metamaterials. The location of the ORB remains invariant with the change of generation order. The width of ORB is determined by the higher frequency band edge for TE polarization and the lower frequency band edge for TM polarization. The location and width of the ORB is not affected by lattice scaling factor. In addition, the defect mode inside the T-M structure is weakly dependent on the incident angle for TE polarization and is affected on incident angle for TM polarization. 


\section{Theoretical Model and Method}

The system under consideration is composed of two layers $A$ and $B$ stacked alternatively along $z$ direction and following the rules of T-M sequence, that is, $S_{n}=S_{n-1} \bar{S}_{n-1}$ with $S_{1}=A B$, here $n$ represents the T-M order and $\bar{S}_{n-1}$ is the complement of $S_{n-1}$ obtained by interchanging $A$ and $B$. The lower order T-M sequences are represented by the strings $S_{1}=A B, S_{2}=A B B A, S_{3}=A B B A B A A B$ and so on. The number of layers in this systems increases with $n$ as $2^{\mathrm{n}}$, while the ratio of the number of layer $A$ to the number of layer $B$ is equal to unity. In this study, layer $A$ and $B$ represent conventional material with thickness $d_{A}$ and IMM with thickness $d_{B}$, respectively. For simplicity, both $\varepsilon_{B}$ and $\mu_{B}$ tensors in the IMM layer are considered to be diagonalizable, i.e.,

$$
\varepsilon_{B}=\left(\begin{array}{ccc}
\varepsilon_{B x} & 0 & 0 \\
0 & \varepsilon_{B y} & 0 \\
0 & 0 & \varepsilon_{B z}
\end{array}\right), \quad \mu_{B}=\left(\begin{array}{ccc}
\mu_{B x} & 0 & 0 \\
0 & \mu_{B y} & 0 \\
0 & 0 & \mu_{B z}
\end{array}\right) .
$$

Without loss of generality, we consider the oblique monochromatic incident wave with the electric field and magnetic field polarized along the y-axis for the transverse electric (TE) and transverse magnetic (TM) wave, respectively. For the TE wave, the electric and magnetic fields can be express as

$$
\begin{aligned}
& E_{B y}=e^{i k_{x} x}\left(C e^{i k_{B z} z}+D e^{-i k_{B z} z}\right) \\
& H_{B x}=\frac{-k_{B z}}{\omega \mu_{0} \mu_{B}} e^{i k_{x} x}\left(C e^{i k_{B z} z}-D e^{-i k_{B z} z}\right) \\
& H_{B z}=\frac{k_{x}}{\omega \mu_{0} \mu_{B}} e^{i k_{x} x}\left(C e^{i k_{B z} z}+D e^{-i k_{B z} z}\right)
\end{aligned}
$$

in the IMM layer, and

$$
\begin{aligned}
& E_{A y}=e^{i k_{x} x}\left(E e^{i k_{A z} z}+F e^{-i k_{A z} z}\right) \\
& H_{A x}=\frac{-k_{A z}}{\omega \mu_{0} \mu_{A}} e^{i k_{x} x}\left(E e^{i k_{A z} z}-F e^{-i k_{A z} z}\right) \\
& H_{A z}=\frac{k_{x}}{\omega \mu_{0} \mu_{A}} e^{i k_{x} x}\left(E e^{i k_{A z} z}+F e^{-i k_{A z} z}\right)
\end{aligned}
$$

in the conventional material layer. $k_{B z}^{2}=\omega^{2} \varepsilon_{B y} \mu_{B x} / c^{2}-\mu_{B x} / \mu_{B z} k_{x}^{2}$ and $k_{A z}^{2}=\omega^{2} \varepsilon_{A} \mu_{A} / c^{2}-k_{x}^{2}$, and $k_{A z}, k_{B z}$ are z-components of wave vector in the conventional material layers and the IMM layers, respectively. $k_{x}$ is the x-component. $C, D, E$ and $F$ are the four constants which are determined by the boundary conditions at the interfaces. For a finite T-M multilayer structure, the electric and magnetic fields from the position $z$ to $z+\Delta z$ in the same layer can be related via a transfer matrix method [3, 7, 8]. The treatment for the TM wave is similar to that for the TE wave, where $k_{B z}^{2}=\omega^{2} \mu_{B y} \varepsilon_{B x} / c^{2}-\varepsilon_{B x} / \varepsilon_{B z} k_{x}^{2}$.

\section{Results and Discussions}

In the following calculation, the Drud model is adopted to describe the tensor components for the permittivity and the permeability of IMM. Such dispersion can be realized in a composite made of periodically LC-loaded transmission lines [7]. $\varepsilon_{B y}=1-100 / \omega^{2}, \mu_{B x}=1.21-100 / \omega^{2}, \mu_{B z}=2$ for TE mode, and $\mu_{B y}=1.21-100 / \omega^{2}, \varepsilon_{B x}=1-100 / \omega^{2}, \varepsilon_{B z}=2$ for TM mode. The operation frequency 
$f=\omega / 2 \pi$ is in units of gigahertz. The permittivity and permeability of layer $A$ are set to be $\varepsilon_{A}=3$ and $\mu_{A}=1$, respectively. The thicknesses are assumed to be $d_{A}=12 \mathrm{~mm}, d_{B}=6 \mathrm{~mm}$. In Fig. 1 , the reflection spectra for normal incidence are shown in case of $S_{4}$ (Fig.1a), $S_{5}$ (Fig.1b), $S_{6}$ (Fig 1c) and $S_{7}$ (Fig. 1d) T-M structures. It can be seen from Fig.1 that, with increasing order of T-M structures, the reflectance bands cease to change and the edges of reflectance band become much sharper. Moreover, very narrow transmission peaks appear and split the reflectance band into two parts at higher frequencies. In fact, the reflectance band can occur in all higher order T-M structures. For simplicity, others are not given in this paper.

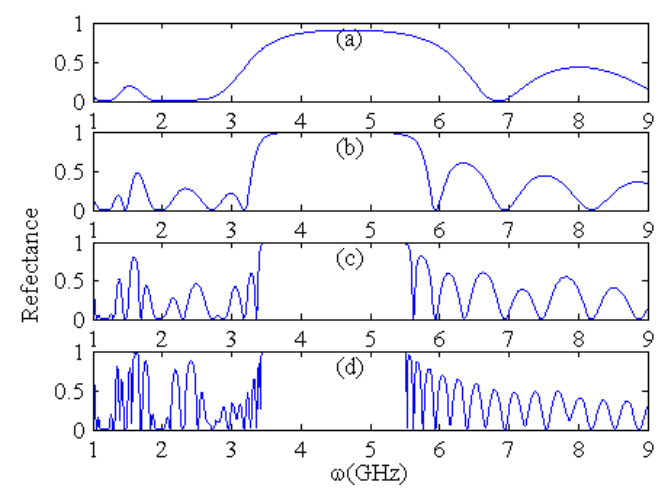

Fig.1 Normal Incidence Reflection Spectra for the Different Order T-M Structures as a Function of the Angular Frequency (a) $S_{4}$, (b) $S_{5}$, (c) $S_{6}$, (d) $S_{7}$

The phenomenon can be explained as follows. In the angle frequency region $\left(0,9 \times 10^{9} \mathrm{GHz}\right)$, $\varepsilon_{B y}$ and $\mu_{B x}$ are simultaneously negative, resulting in a negative refractive in IMM. Equation $k_{A z} d_{A}+k_{B z} d_{B}=0$ can be implemented, which indicates that the zero- $\bar{n}$ gap exists. This condition is similar to the one dimension photonic crystal containing isotropic negative-index materials [8]. The TM wave can be analyzed in a similar way.

In Fig.2, we show reflectance for both TE (the right part) and TM (the left part) polarizations versus angular frequency for different incident angle. We take $S_{6}$ as an example and investigate the wide angle reflectance behavior of the systems. It is illustrated that the higher band edge of reflection band is insensitive to the increase of incident angle and the lower band edge shift towards high frequency for TE polarization, but the case for TM polarization is different, the lower band edge nearly keep constant and the higher band edge shift towards high frequency. So the frequency range of the omnidirectional reflectance band for both polarizations is between $4 \mathrm{GHz}$ and $5.2 \mathrm{GHz}$, which is determined by the higher band edge for TE polarization and the lower band edge for TM polarization. This property is similar to omnidirectional reflection band coming from zero- $\bar{n}$ gap mechanisms in one dimensional photonic crystal containing anisotropic left-handed materials [8]. But the property is different fundamentally from that of omnidirectional reflection band in dielectric T-M structures [13], in which omnidirectional reflection band depended only on TM polarization.

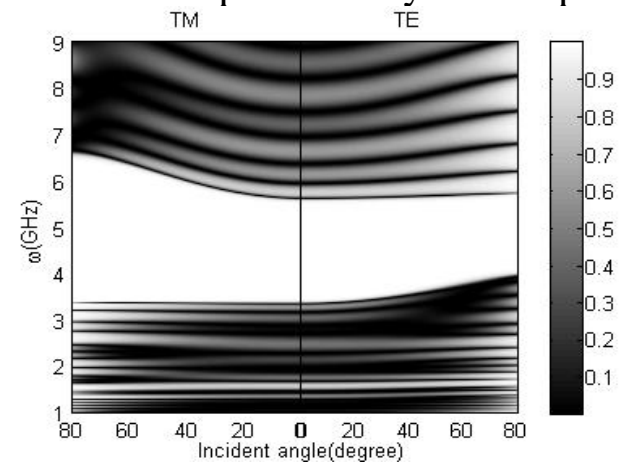

Fig.2 Dependence of TM (the Left Part) and TE (the Right Part) Polarization Reflection Spectra for the $\mathrm{S}_{6} \mathrm{~T}-\mathrm{M}$ Structure 
To study the influence of size scaling of the T-M structure, a scaling factor $\rho=d / d_{0}$ is defined, where $d_{0}$ indicates the thickness $\left(\mathrm{d}_{1}=12 \mathrm{~mm}, \mathrm{~d}_{2}=6 \mathrm{~mm}\right)$ before scaling and $d$ indicates the thickness after scaling. The reflection spectra of $\mathrm{S}_{6}$ structure at different scaling factor is shown in Fig.3 (for TE polarization) and Fig.4 (for TM polarization). It is apparent that the center of reflection band almost remains at the same frequency, and the two band edges are nearly invariant for both TE and TM polarizations as the scaling factor $\rho$ increases. Such results indicate that there is an omnidirectional gap coming from the zero- $\bar{n}$ gap mechanism [8].

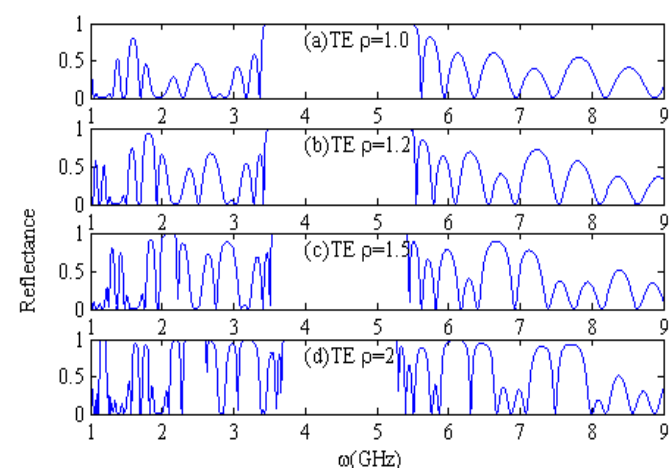

Fig.3 The Reflection Spectra of $\mathrm{S}_{6}$ Structure at Different Scaling Factor for TE Polarization

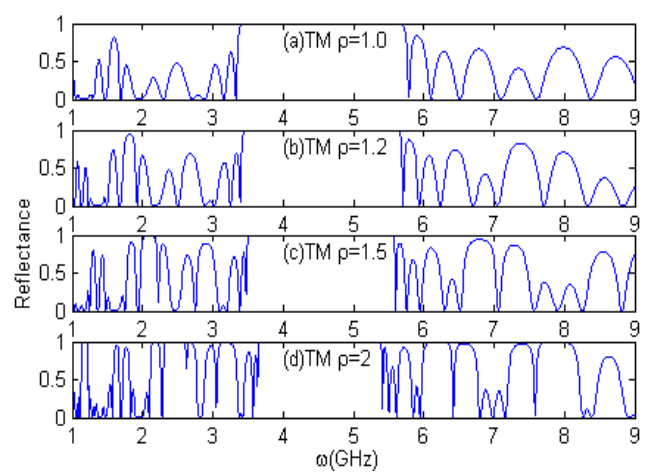

Fig.4 The Reflection Spectra of $\mathrm{S}_{6}$ Structure at Different Scaling Factor for TM Polarization

Next we consider the case where the localized defect mode is induced by a defect $\mathrm{Z}$ with $\varepsilon_{z}=2.5$, $\mu_{z}=1$ and $\mathrm{d}_{\mathrm{z}}=4 \mathrm{~mm}$ inside the T-M structure with $\mathrm{S}_{6} \mathrm{CS}_{6}$. In Fig.5, it explicitly reveals that the defect mode inside the zero- $\bar{n}$ gap is weakly affected with the incident angles increases from $0^{\circ}$ to $80^{\circ}$ for TE polarizations. However, figure 6 shows that defect mode is strongly affected when the incident angle from 0 to 80 for TM polarizations. Such properties is different from the reflection band coming from zero- $\bar{n}$ gap mechanisms in one dimensional photonic crystal containing anisotropic left-handed materials $[7,8]$.

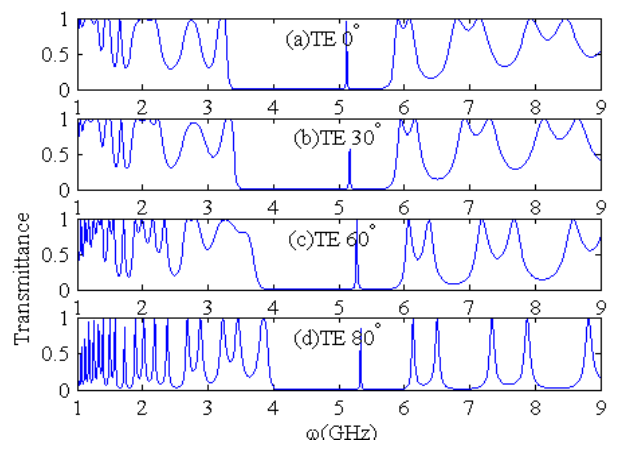

Fig.5 Transmission Spectra of the T-M Structure with a Defect Z for TE Polarization

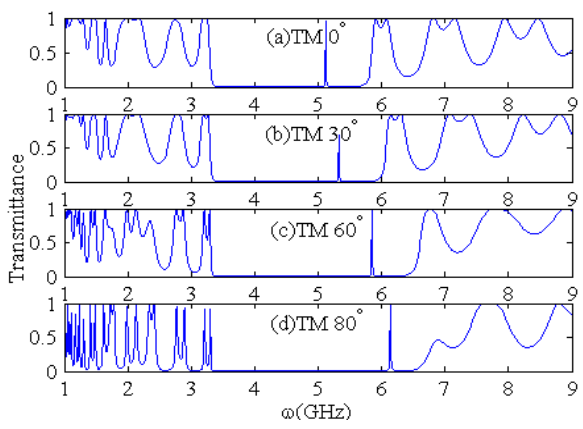

Fig.6 Transmission Spectra of the T-M Structure with a Defect Z for TM Polarization 
Finally it should be pointed out that the loss of IMM cannot be neglected though the imaginary parts of permittivity and permeability at some frequency are very low. In our calculation, it is found that the band edges of the omnidirectional gap and the defect mode are not affected when a loss is considered, since the loss only affects the magnitude of transmission [10].

\section{Conclusion}

In summary, the ORB of one dimensional quasicrystal T-M structure composed by conventional material $A$ and IMM $B$ have been studied. We have proven that ORB cease to change with the increase of generation order. The width of the ORB is determined by the higher band edge for TE polarization and the lower band edge for TM polarization. The location and width of the ORB is not affected by lattice ratio factor. In addition, the defect mode inside the T-M structure is weakly dependent on the incident angle for TE polarization and is affected on incident angle for TM polarization.

\section{Acknowledgement}

This research was financially supported by the National Science Foundation (Grant NO.61307121) and Launching Scientific Research Funds for Doctors (Grant NO.2014-B-04).

\section{References}

[1] D. R. Smith, D. Schurig, "Electromagnetic Wave Propagation in Media with Indefinite Permittivity and Permeability Tensors," Phys. Rev. Lett. 90 (2003) 077405.

[2] C.L. Cirtes, W. Newman, S. Molesky, Z. Jacob, "Quantum nanophotonics using hyperbolic metamaterials," J. Opt. 14 (2012) 063001.

[3] Y.Q. Kang, C.M. Zhang, C.H. Xue, Q.Z. Cao, W.Y. Ren, "Wannier stark ladder in one-dimensional photonic crystal coupled microcavity containing indefinite metamaterials," J. Opt. 42(4) (2013) 335-340.

[4] D. R. Smith, P. Kolinko, D. Schurig, "Negative refraction in indefinite media," J. Opt. Soc. Am. B 21 (2004)1032-1043.

[5] D. R. Smith, D. Schurig, J. J. Mock, P. Kolinko, P. Rye, "Partial focusing of radiation by a slab of indefinite media," Appl. Phys. Lett. 84 (2004) 2244.

[6] L.B. Hu, S. T. Chui, "Characteristics of electromagnetic wave propagation in uniaxially anisotropic left-handed materials,” Phys. Rev. B 66085108 (2002).

[7] Y. J. Xiang, X. Y. Dai, S. C. Wen, D. Y. Fan, "Properties of omnidirectional gap and defect mode of one-dimensional photonic crystal containing indefinite metamaterials with a hyperbolic dispersion,” J. Appl. Phys. 102 (2007) 093107.

[8] S. Wang, L. Gao, "Omnidirectional reflection from the one-dimensional photonic crystal containing anisotropic left-handed material,” Eur. Phys. J. B 48 (2005) 29-36.

[9] L. W. Zhang, K. Fang, G. Q. Du, H. T. Jiang, J. F. Zhao, "Transmission properties of Fibonacci quasi-periodic one-dimensional photonic crystals containing indefinite metamaterials," Opt. Communications 284 (2011) 703-706.

[10] Y.Q. Kang, C.M. Zhang, T.K. Mu, P. Gao "Resonant modes and inter-well coupling in photonic double quantum well structures with single-negative materials," Opt. Commun. 285(24) (2012) 4821-4824.

[11]Quasicrystals, edited by T. Fujiwara, T. Ogawa (Springer, Heidelberg, 1990). 
[12] X. Jiang, Y. Zhang, S. Feng, K.C. Huang, Y. Yi, J.D. Joannopoulos, "Photonic band gaps and localization in the Thue-Morse structures,"Appl. Phys. Lett. 86 (2005) 201110.

[13] L.D. Negro, M. Stolfi, Y. Yi, J. Michel, X. Duan, L.C. Kimerling, J. LeBlanc, J. Haavisto, "Photon band gap properties and omnidirectional reflectance in SiSiO2 Thue-Morse quasicrystals" Appl. Phys. Lett. 84 (2004) 5186-5188.

[14] L. Moretti, I. Rea, L. Rotiroti, I. Rendina, G. Abbate, A. Marino, L.De. Stefano, "Photonic band gaps analysis of Thue-Morse multilayers made of porous silicon," Opt. Express 14 (2006) 6264.

[15] C.P. Yin, T.B. Wang, J.W. Dong, Y.H. Chen, H.Z. Wang, "Omnidirectional reflection and flat-top transmissionin Thue-Morse quasicrystal with single-negative materials," Eur. Phys. J. B 69 (2009) 357-361. 